\title{
Influência dos aspectos motivacionais sobre as respostas afetivas após teste de esforço máximo
}

\section{The influence of motivational aspects in the Affective Response after a maximum effort test}

La influencia de los aspectos motivacionales en la respuesta afectiva después de una prueba de esfuerzo máximo

\author{
Vanessa Pessota \\ Centro de Estudos em Psicobiologia e Exercício (CEPE), São Paulo, Brasil \\ Amaury Tavares Barreto \\ Programa de Pós-graduação em Nutrição, Universidade Federal de São Paulo, Unifesp, São Paulo-SP, Brasil \\ Marco Túlio De Mello \\ Departamento de Psicobiologia, Universidade Federal de São Paulo, UNIFESP, São Paulo-SP, Brasil \\ Hanna Karen M. Antunes ${ }^{1}$ \\ Departamento de Biociências, Universidade Federal de São Paulo, UNIFESP, Santos-SP, Brasil
}

\begin{abstract}
Resumo
Sete voluntários foram submetidos a três protocolos idênticos de exercício físico com cargas progressivas realizados até a exaustão voluntária máxima nas condições de Encorajamento verbal, Silêncio e Música para se investigar suas respostas afetivas. Em todos os protocolos, os voluntários responderam aos seguintes instrumentos: BRUMS; VAMS; SEES e IDATE nos momentos basal (antes da realização do teste físico), imediatamente após cada um dos protocolos de exercício, 30' e 60' após o término do exercício. Observaram-se mudanças evidentes nos grupos em relação ao tempo particularmente nas variáveis psicobiológicas (humor e bem-estar), porém não foram observadas mudanças nas variáveis fisiológicas estudadas. Desta forma, pode-se concluir que os aspectos psicobiológicos parecem ser mais sensíveis a modificações do que as variáveis fisiológicas quando em situação de exercício máximo. Palavras chave: Humor; Motivação; Exercício Físico; Música.
\end{abstract}

\begin{abstract}
Seven subjects underwent three exercise protocols with identical loads progress to the maximum voluntary exhaustion under conditions of verbal encouragement, Music and Silence to investigate their affective responses. In all protocols, the volunteers answered to the following instruments: BRUMS; VAMS, STAI and SEES in different time-course: baseline (prior to the exercise protocol), immediately after each exercise protocols, 30' and 60' after the end of the protocol. Changes have been evident in the groups related to time course particularly in psychobiological variables (mood and well-being), but there were no changes in physiological variables. Thus, we can conclude that the psychobiological aspects seem to be more sensitive to changes than physiological variables in maximum exercise.

Keywords: Mood; Motivation; Physical Exercise; Music.
\end{abstract}

\section{Resumen}

Siete voluntarios fueron sometidos a tres protocolos idénticos de ejercicio físico con cargas progresivas llevados a cabo hasta el agotamiento voluntario máximo en las condiciones de Encorajamiento verbal, Silencio y Música para investigar sus respuestas afectivas. En todos los protocolos, los voluntarios respondieron a los siguientes instrumentos: BRUMS; VAMS; SEES e IDATE en momento basal (antes de llevar a cabo la prueba física), inmediatamente después de cada uno de los protocolos de ejercicio, 30' y 60' después del término de ellos. Se observaran cambios evidentes en los grupos en relación al tiempo particularmente en las variables psicobiológicas (humor y bien-estar), entretanto no fueron observados cambios en las variables fisiológicas estudiadas. De esta forma, se puede concluir que los aspectos psicobiológicos parecen ser más sensibles a modificaciones de que las variables fisiológicas cuando nos encontramos en situación de ejercicio máximo.

Palabras clave: Humor; Motivación; Ejercicio Físico; Música.

\section{Introdução}

O desempenho físico é uma resultante de diferentes fatores, dentre eles, aspectos genéticos, anos de treinamento, domínio técnico do movimento,

1 Endereço:Centro de Estudos em Psicobiologia e Exercício-

CEPE - Rua: Professor Francisco de Castro, 93 Vila

Clementino São Paulo- SP Cep: 04020-050

e-mail: hanna.karen@unifesp.br aspectos nutricionais (Rogol, 2003). No sentido de aprimorar cada um desses elementos, muitas vezes, técnicos e atletas utilizam de recursos pouco convencionais, algumas vezes proibidos, mas com certeza, prejudiciais ao organismo humano como, por exemplo, recursos ergogênicos. Por outro lado, outras estratégias que não prejudicam o organismo, como a música, podem atuar como elementos reforçadores e representar a linha tênue que separa a frustração da 
fama e a glória esportiva.

A música tem sido utilizada como elemento motivacional no sentido de elevar a performance esportiva (Copeland \& Franks, 1991) e tem se mostrado como um grande atrativo para pesquisadores (Karageoghis \& Terry, 1997). Os argumentos para o uso da música são diversos, dentre eles, que o esforço seria menos monótono e árduo, resultando em um maior esforço e maior aderência, o que influenciaria de forma positiva o desempenho físico. Assim, o seu uso permitiria um controle do ritmo de execução do exercício, aumentando os elementos emotivos e distrativos em atletas (Szmedra \& Bacharach, 1988; Szabo, Small \& Leigh, 1999), o que coincide com os frequentes relatos de seus efeitos favoráveis, seja durante o treinamento e/ou competição. Embora pareça interessante, o uso da música no contexto esportivo tem recebido relativamente pouca atenção. Em parte, isso acontece devido às poucas evidências científicas acerca do tema, sugerindo que muitas vezes os resultados existentes foram dependentes de parâmetros como, por exemplo, tempo e tipo de música, tipo de exercício empregado e condicionamento físico da amostra (Becker, et al.,1994; Pujol \& Langenfeld, 1999).

De forma geral, escutar música enquanto se exercita, aumenta a carga de trabalho, e os efeitos psicofísicos estão relacionados com redução da percepção subjetiva de esforço e aumento dos estados afetivos (Karageorghis, Terry \& Lane, 1999) o que pode dar a essa estratégia um caráter de recurso ergogênico lícito e benéfico.

Embora seja discutível, o mecanismo pelo qual a música pode influenciar a performance atlética parece estar relacionada com aumento do nível do estado de alerta, com decréscimo da fadiga e aumento da sincronização e coordenação motora (Karageoghis \& Terry, 1997). Nesse sentido, muitos pesquisadores têm encontrado efeitos benéficos da música no desempenho atlético, com efeitos relaxantes de músicas lentas (Ferguson, Carbonneau \& Chambliss, 1994; Karageorghis, Drew \& Terry, 1996), sugerindo que esse tipo de música pode ser um determinante na capacidade de exercício. Szabo, Small \& Leigh (1999), observaram que o uso de música lenta progressiva a música rápida durante um exercício incremental resulta no alcance de cargas mais elevadas. Becker et al. (1994), demonstrou que tanto a música rápida quanto lenta melhora o desempenho supra máximo em crianças e adultos jovens. Por outro lado, Copeland \& Franks (1991), encontraram que a música lenta apresenta benefícios em respostas fisiológicas e psicológicas comparáveis ao alerta produzido pela música em exercícios sub-máximos.

Parece bastante coerente que a seleção das músicas represente uma tentativa de otimizar o alcance da meta do atleta. Quando o atleta precisa ser motivado ou permanecer alerta para executar uma performance supra máxima, potência ou tarefa muscular, uma música rápida e despertante precisa ser utilizada. O uso desse mesmo tipo de música pode prejudicar atletas cujo desempenho esteja relacionado com concentração e coordenação motora (Eliakim, Meckel, Nemet \& Eliakim, 2007).

Escutar música rápida durante o aquecimento resulta em um aumento significativo na freqüência cardíaca durante os primeiros minutos de protocolos de multi-estágio em esteira (Copeland \& Franks, 1991). Yamamoto et al. (2003) mostrou que escutar música rápida promove um aumento nos níveis plasmáticos de epinefrina, enquanto escutar música lenta diminui esses níveis previamente a exercício submáximo. Esses resultados permitem diversas especulações, e com certeza elas podem auxiliar na compreensão de elementos que podem contribuir com o desempenho de um atleta. É importante mencionar que a percepção do esforço está relacionada principalmente com intensidade e duração do exercício. Durante o exercício supra-máximo, atletas expressam seus sentimentos que variam de fadiga geral até exaustão.

Escutar música distrai o atleta de sensações de fadiga associados ao exercício, o que é chamado de modelo de processamento paralelo (Rejeski, 1985). Isso sugere que esse mecanismo é influenciado principalmente durante baixas intensidades de exercício, quando sinais externos podem competir com sinais internos. Durante altas intensidades, sinais internos como fadiga apresentam um forte impacto no status mental. Nesse sentido, elementos distratores como a música, podem promover uma melhora das capacidades físicas de um indivíduo submetido a um teste de esforço máximo voluntário e parte dessa melhora pode estar relacionada com o humor e a motivação pelo exercício físico em si.

Assim, o objetivo do presente estudo foi o de investigar as respostas afetivas durante o exercício físico realizado até a exaustão voluntária máxima quando esse é realizado a partir de diferentes tipos de incentivo.

\section{Metodologia}

\section{Descrição da amostra}

Participaram deste estudo 07 voluntários jovens, hígidos do sexo masculino com média \pm desvio padrão da idade, massa corporal total, estatura e índice de massa corporal de: $32,76 \pm 8,87$ anos; $75,74 \pm 7,17 \mathrm{Kg} ; 1,75 \pm 0,04 \mathrm{~m}$; e $24,45 \pm 1,91$ $\mathrm{Kg} / \mathrm{m} 2$, respectivamente. Para atestar a saúde cardiovascular, os voluntários foram submetidos a um Eletrocardiograma de repouso (ECG) e a um Teste Ergométrico. Aqueles considerados aptos pelo médico foram inseridos no estudo. Os voluntários foram submetidos a três protocolos idênticos de carga progressiva até a exaustão voluntária máxima. Em cada um deles, foi determinado o consumo pico 
de oxigênio e dos limiares ventilatórios, bem como as demais variáveis relacionadas a essa avaliação. Para cada tentativa, foi dado um diferente tipo de estímulo durante o teste, a saber: 1) Verbal - os voluntários obtiveram incentivos verbais durante todo o período do protocolo, sendo usadas frases positivas como: "muito bom", "continua assim", "vamos lá", “está ótimo", "excelente", e outros; 2) Silêncio - os voluntários foram apenas informados sobre início e o final do teste, e questionados se estavam bem. Nenhum estímulo verbal foi dado; 3) Música - os voluntários escutaram uma música durante o teste. A música foi montada com duração de 16 minutos com auxílio do programa MixMeister Fusion versão 7.2. Ao todo, foram utilizadas 04 músicas, cujos trechos foram retirados e montados na seguinte seqüência: 1-The Shapeshifters (Lola's Theme); 2-Infected Mushroom (Become Insane); 3-Infected Mushroom (Merlin); 4-Infected Mushroom (Extacy). A batida da música foi iniciada em ritmo mais lento $110 \mathrm{bpm}$ e aumentada gradativamente até atingir $210 \mathrm{bpm}$. Durante a realização desse protocolo, a música foi reproduzida aos voluntários pelo programa Itunes $\AA$ versão 9.1.0.79 Copyright 2000-2010 Apple Inc. ${ }^{\circledR}$, utilizando um notebook CCE® modelo Core2duo XLE-432 ${ }^{2}$ 4GB/320HD com um fone de ouvido modelo Shuffle Ipod ${ }^{\circledR}$, sendo o volume fixado em $90 \%$ durante todo o teste. Todos os procedimentos utilizados nesse estudo foram aprovados pelo Comitê de Ética da Universidade Federal de São Paulo/ Hospital São Paulo-UNIFESP e aprovado sob o número \#1629/08.

\section{Descrição dos protocolos experimentais} Exercício:

a) Carga Progressiva até a exaustão voluntária máxima:

O teste de carga progressiva em um cicloergômetro de membro inferior foi conduzido em uma bicicleta modelo Lode Excalibur (Lode, Excalibur Sport 925900; Groningen, Netherlands). O protocolo adotado para o teste foi o de incrementos de 35 watts a cada 2 minutos com uma carga inicial de aquecimento fixada em 70 watts. Durante todo o teste foi solicitado ao voluntário manter a cadência de 70 RPM, sendo o teste encerrado ao atingir a exaustão voluntária máxima. A Exaustão voluntária máxima foi concebida pela inabilidade do voluntário em manter o ritmo cadenciado (pedalando em uma frequência abaixo de 70 RPM sem possibilidade de recuperação e retorno a cadência estipulada em até 15 segundos), ou até que os mesmos parassem de se exercitar espontaneamente (Sassi, Marcora, Rampinini, Mognoni \& Impellizzeri, 2006). Para essa avaliação, foi realizada uma análise dos gases ventilatórios, para determinar as seguintes variáveis respiratórias: consumo pico de oxigênio ( $\mathrm{O} 2$ pico), limiar ventilatório I (LV-I), limiar ventilatório II (LV-II), freqüência cardíaca máxima
(FCMÁX), frequência cardíaca do limiar ventilatório I (FCLV-I), frequência cardíaca do limiar ventilatório II (FCLV-II), e carga na intensidade do Limiar I e II. Para a determinação do consumo de oxigênio no limiar ventilatório I e II (LV-I e LV-II), foram observados os critérios descritos por Wasserman (1987) e Wasserman \& Koike (1992). Essas variáveis foram obtidas pelo método de mensuração das trocas gasosas respiratórias com um sistema metabólico (Quark, PFT - Pulmonary Function Testing - FRC \& DLCO- 4ergo; Cosmed, Italy). O sistema foi calibrado antes da realização de cada protocolo, usando uma concentração de gases conhecidos, sendo que as calibrações do volume e do fluxo foram realizadas com o auxílio de uma seringa de três litros. Uma máscara facial Hans Rudolph ${ }^{\circledR}$ flow-by face mask (Kansas City, MO, EUA), foi utilizada. Durante todos os testes, a monitoração da freqüência cardíaca foi realizada por meio de um frequencímetro (Polar ${ }^{\circledR}$, modelo S810i) com intervalos de 20 segundos. Os testes foram realizados em laboratório com climatização padronizada e no mesmo período do dia.

Para evitar uma possível interferência, não foi permitido aos voluntários acompanhar o comportamento de seus parâmetros fisiológicos durante o teste (FC, O2, velocidade, carga, etc). Os protocolos foram realizados no mesmo horário do dia e separados por um período de washout mínimo de $48 \mathrm{~h}$ e máximo de sete dias. Algumas recomendações foram dadas aos voluntários referentes a não ingerir alimentos e bebidas $2 \mathrm{~h}$ antes do teste, com exceção de água, e não fazer exercício $24 \mathrm{~h}$ antes de cada teste.

\section{Humor}

Para cada protocolo de exaustão, os voluntários responderam a três instrumentos que avaliam respostas afetivas e aspectos do bem-estar. Essa avaliação foi realizada em diferentes momentos: basal, imediatamente após cada um dos protocolos de exercício, 30' e 60' após o término dos protocolos. Os instrumentos utilizados foram:

1) Escala de Humor de Brunel (BRUMS) Desenvolvida para medir rapidamente o estado de humor por Terry, Lane \& Forgaty (2003), foi adaptado do "Profile of Mood States" (POMS) (Mcnair, Lorr \& Droppleman, 1971) e traduzida para o português por Rohlfs , Carvalho \& Rotta (2004). Consiste em uma lista com 24 adjetivos relacionados ao estado de humor, onde o avaliado deve anotar como se sente em relação a cada adjetivo, conforme as instruções considerando uma escala tipo Likert de 0 a 4 . Seis fatores de humor ou estados afetivos são medidos por esse instrumento: tensão, depressão, raiva, vigor, fadiga e confusão. É esperado nesse teste que os valores encontrados para a dimensão vigor sejam maiores que os valores apresentados nas outras dimensões, o que denotaria um perfil de humor em forma de "Iceberg". 
Tabela 1

Descritiva dos resultados do teste ergoespirométrico realizado até a exaustão voluntária máxima

\begin{tabular}{|c|c|c|c|}
\hline Variáveis & Verbal & Silêncio & Música \\
\hline $\mathrm{VO}_{2}$ pico $\left(\mathrm{L} \cdot \mathrm{min}^{-1}\right)$ & $3,89 \pm 0,96$ & $3,66 \pm 0,71$ & $3,73 \pm 0,76$ \\
\hline $\mathrm{VO}_{2}$ pico $\left(\mathrm{mL} \cdot \mathrm{kg} \cdot \mathrm{min}^{-1}\right)$ & $50,88 \pm 9,19$ & $48,04 \pm 7,90$ & $48,41 \pm 8,04$ \\
\hline FC Max (bpm) & $188,14 \pm 15,36$ & $184,14 \pm 12,46$ & $184,43 \pm 12,34$ \\
\hline VE Max (L) & $153,14 \pm 24,27$ & $141,49 \pm 27,23$ & $149,39 \pm 19,43$ \\
\hline Tempo max. (min) & $17,54 \pm 4,07$ & $16,77 \pm 4,75$ & $16,57 \pm 4,33$ \\
\hline $\mathrm{VO}_{2}$ LVI $\left(\mathrm{L} \cdot \mathrm{min}^{-1}\right)$ & $2,76 \pm 0,67$ & $2,63 \pm 0,83$ & $2,71 \pm 0,73$ \\
\hline $\mathrm{VO}_{2} \mathrm{LVI}\left(\mathrm{mL} \cdot \mathrm{kg} \cdot \mathrm{min}^{-1}\right)$ & $36,13 \pm 6,75$ & $34,37 \pm 9,44$ & $34,95 \pm 8,00$ \\
\hline FC LVI (bpm) & $151,14 \pm 16,41$ & $144,71 \pm 15,28$ & $151,14 \pm 16,43$ \\
\hline Carga LVI (watts) & $215,00 \pm 47,08$ & $201,00 \pm 71,12$ & $215,00 \pm 76,76$ \\
\hline Tempo LVI (min) & $10,69 \pm 2,95$ & $9,69 \pm 3,80$ & $9,71 \pm 3,91$ \\
\hline $\mathrm{VO}_{2}$ LVII $\left(\mathrm{L} \cdot \mathrm{min}^{-1}\right)$ & $3,24 \pm 0,74$ & $3,11 \pm 0,75$ & $3,17 \pm 0,64$ \\
\hline $\mathrm{VO}_{2} \mathrm{LVII}\left(\mathrm{mL} \cdot \mathrm{kg} \cdot \mathrm{min}^{-1}\right)$ & $42,47 \pm 7,63$ & $40,81 \pm 8,67$ & $41,12 \pm 6,72$ \\
\hline FC LVII (bpm) & $172,00 \pm 16,51$ & $163,71 \pm 12,04$ & $169,00 \pm 12,26$ \\
\hline Carga LVII (watts) & $265,00 \pm 66,58$ & $245,00 \pm 63,90$ & $260,00 \pm 66,58$ \\
\hline Tempo LVII (min) & $13,71 \pm 3,67$ & $12,77 \pm 3,81$ & $12,57 \pm 3,18$ \\
\hline
\end{tabular}

Dados apresentados em média \pm desvio-padrão. Legenda: VO2- consumo de oxigênio; FC- freqüência cardíaca; VE- ventilação; LVI- Limiar ventilatório 1; LVII- Limiar ventilatório 2. Não foram observadas diferenças significativas entre os grupos.

2) Visual Analogues of Mood Scales (VAMS) 16 escalas analógicas de $100 \mathrm{~mm}$ através das quais o avaliado por meio de um traço vertical avalia as alterações de humor (Bond, Lader, 1974; Guimarães, Zuardi, Gentil \& Graeff, 1998).

3) Escala Subjetiva de Experiência em ExercícioSEES (Mcauley \& Courneya, 1994) - Instrumento utilizado para observar respostas afetivas induzidas pelo exercício físico. Trata-se de uma escala tridimensional, que avalia as seguintes dimensões: (a) bem-estar positivo; (b) distresse psicológico e (c) fadiga. A principal questão é: "Como você se sente agora?", a escala é composta por 12 itens graduados em uma escala tipo Likert que varia de 1 a 7, onde 1 significa nenhum pouco e 7 significa muitíssimo (Mcauley \& Courneya, 1994; Lox \& Rudolph, 1994).

4) Idate - É um questionário de auto-avaliação que avalia a ansiedade. Está dividido em duas partes: uma avalia a ansiedade-traço (referindo-se a aspectos de personalidade) e a segunda avalia a ansiedade-estado (referindo-se a aspectos sistêmicos do contexto). Cada uma dessas partes é composta de 20 afirmações. Ao responder o questionário, o indivíduo deve levar em consideração uma escala de quatro itens que variam de 1 a 4, sendo que ESTADO significa como o sujeito se sente no "momento" e TRAÇO como ele "geralmente se sente". O escore de cada parte varia de 20 a 80 pontos, sendo que os escores podem indicar um baixo grau de ansiedade (0-30), um grau mediano de ansiedade (entre 31 e 49) e um grau elevado de ansiedade (maior ou igual a 50), quanto mais baixo se apresentarem os escores, menor será o grau de ansiedade (Spielberg, Gorshusch \& Lushene, 1970; Biaggio \& Natalicio, 1979; Andreatini \& Seabra, 1993). Nesse estudo, a parte referente à ansiedade Traço foi aplicada em um único momento, por se tratar do traço de personalidade do voluntário, já para o estado de ansiedade foi aplicado de acordo com os diferentes momentos previamente descritos.

\section{Análise estatística}

Os dados colhidos foram analisados conforme o comportamento das variáveis após a realização do teste de normalidade Shapiro Wilk's. Em um primeiro momento foi feita uma análise descritiva dos dados e posteriormente, foi aplicado uma análise de variância ANOVA para medidas repetidas, usando como fatores TEMPO e GRUPO e post-hoc Duncan Test. O nível de significância considerado em todas as análises foi de $p<0,05$. As análises estatísticas foram conduzidas com auxílio do pacote estatístico Statistica ${ }^{\circledR}$ versão 7.0.

\section{Resultados}

Na tabela 1 é apresentada a análise descritiva dos resultados do teste ergoespirométrico realizado até a exaustão voluntária máxima. Quando os diferentes protocolos foram comparados não foram encontradas diferenças significativas entre eles. $\mathrm{Na}$ tabela 2 são apresentados os resultados referentes à Escala Subjetiva de Experiência em Exercício SEES nos diferentes grupos de estudo. Quando as 
Tabela 2

Resultados da Escala Subjetiva de Experiência em Exercício SEES- nos diferentes grupos.

\begin{tabular}{llcccc}
\hline Dimensões & Grupos & Basal & $\begin{array}{c}\text { Imediatamente } \\
\text { após }\end{array}$ & $30{ }^{\prime}$ & $60^{\prime}$ \\
\hline \multirow{2}{*}{$\begin{array}{l}\text { Bem-estar } \\
\text { positivo }\end{array}$} & Verbal & $16,57 \pm 1,90$ & $14,29 \pm 5,41 \mathrm{a}$ & $17,57 \pm 20,00 \mathrm{~b}$ & $16,71 \pm 3,25 \mathrm{~b}$ \\
& Silêncio & $16,57 \pm 2,82$ & $13,86 \pm 2,04 \mathrm{a}$ & $15,29 \pm 3,20$ & $15,14 \pm 3,13$ \\
& Música & $17,43 \pm 2,82$ & $14,43 \pm 2,51 \mathrm{a}$ & $16,14 \pm 2,54$ & $16,29 \pm 4,23$ \\
\hline \multirow{2}{*}{$\begin{array}{l}\text { Distresse } \\
\text { Psicológico }\end{array}$} & Verbal & $4,86 \pm 1,07$ & $6,57 \pm 3,99 \mathrm{a}$ & $5,43 \pm 9,00$ & $4,43 \pm 0,79 \mathrm{~b}$ \\
& Silêncio & $4,57 \pm 0,53$ & $6,29 \pm 1,98 \mathrm{a}$ & $5,00 \pm 0,58$ & $4,43 \pm 0,53 \mathrm{~b}$ \\
\hline \multirow{2}{*}{ Fadiga } & Música & $4,29 \pm 0,49$ & $4,86 \pm 0,69$ & $4,43 \pm 0,79$ & $4,57 \pm 0,53$ \\
\hline & Silêncio & $9,14 \pm 5,27$ & $18,86 \pm 4,91 \mathrm{a}$ & $12,43 \pm, 99 \mathrm{~b}$ & $11,00 \pm 4,40 \mathrm{~b}$
\end{tabular}

ANOVA two-way para medidas repetidas com fatores Tempo e Grupo, com post-hoc utilizando Duncan Test. $\mathrm{a}$ - diferente do basal; $\mathrm{b}$ - diferente do imediatamente após. Resultados significativos para $\mathrm{p} \leq 0,05$. Dados apresentados em média \pm desvio-padrão.

análises foram realizadas, encontramos diferenças significativas apenas para o fator tempo, onde o momento imediatamente após foi diferente do momento basal em todas as dimensões e em todos os grupos, com exceção do grupo música na dimensão distresse psicológico. Considerando os momentos que se seguiram ao teste, apenas a dimensão fadiga continuou apresentando alterações seja comparado ao basal ou ao momento imediatamente nos 3 protocolos de estudo. As outras dimensões se mostraram distintas, mas não para todos os grupos. Na tabela 3 são apresentados os resultados referentes ao questionário Idate Estado. Os três grupos esboçaram comportamentos similares onde observamos uma resposta ansiogênica do exercício na condição imediatamente após seguido de um comportamento de redução desses escores nos momentos 30' e 60'. Nas outras análises não foram encontradas diferenças significativas. $\mathrm{Na}$ tabela 4 são apresentados os resultados referentes ao questionário de perfil de humor de Brunel. Na condição imediatamente após, as dimensões DTH e Fadiga apresentaram aumentos em todos os grupos quando comparado com o basal. Nas dimensões Depressão, Fadiga e DTH observamos uma diminuição desses escores em 30 e 60'quando comparados a condição basal, sugerindo uma recuperação parcial e total respectivamente para os grupos estudados. Na comparação entre os grupos observamos menores escores no grupo Silêncio e Música quando comparado ao grupo Verbal em relação à dimensão Depressão observada 30'após o exercício e nas dimensões Confusão Mental e o DTH, as quais apresentaram comportamento similar, onde o grupo música apresentou menores escores quando comparado ao grupo Verbal nas condições de 30 e 60 minutos. Na figura 1 são apresentados os resultados do VAMS. Não encontramos diferenças quando os grupos foram comparados, no entanto em relação ao tempo, observamos que no grupo que foi submetido ao exercício com encorajamento verbal, na dimensão sedação física houve uma diminuição no momento imediatamente após e na dimensão sedação mental, 60 minutos após o exercício, observamos diminuição dos escores em relação ao

TABELA 3

Resultados do questionário Idate Estado nos diferentes grupos

\begin{tabular}{lllcc}
\hline Protocolos & Basal & Imediatamente após & $30^{\prime}$ & $60^{\prime}$ \\
\hline Verbal & $33,86 \pm 2,73$ & $40,00 \pm 8,94 \mathrm{a}$ & $33,71 \pm 5,12 \mathrm{~b}$ & $30,00 \pm 2,89 \mathrm{~b}$ \\
Silêncio & $34,29 \pm 4,64$ & $40,29 \pm 8,28 \mathrm{a}$ & $31,29 \pm 3,45 \mathrm{~b}$ & $29,71 \pm 5,35 \mathrm{~b}$ \\
Música & $33,29 \pm 3,77$ & $42,43 \pm 6,00 \mathrm{a}$ & $33,57 \pm 3,78 \mathrm{~b}$ & $31,71 \pm 5,44 \mathrm{~b}$ \\
\hline
\end{tabular}

ANOVA para medidas repetidas, com post-hoc utilizando Duncan Test. a - diferente do basal intra grupo; $\mathrm{b}$ - diferente do imediatamente após intra grupo. Resultados significativos para $\mathrm{p} \leq 0,05$. Dados apresentados em média \pm desvio-padrão. 
Tabela 4

Resultados do questionário de perfil de humor-BRUMS

\begin{tabular}{|c|c|c|c|c|c|c|}
\hline \multirow{2}{*}{ Variáveis } & \multicolumn{3}{|c|}{ Basal } & \multicolumn{3}{|c|}{ Imediatamente após } \\
\hline & Verbal & Silêncio & Música & Verbal & Silêncio & Música \\
\hline $\begin{array}{l}\text { Tensão- } \\
\text { Ansiedade }\end{array}$ & $2,00 \pm 1,41$ & $2,29 \pm 1,89$ & $1,86 \pm 0,69$ & $2,29 \pm 2,93$ & $2,57 \pm 1,72$ & $2,71 \pm 3,15$ \\
\hline Depressão & $1,14 \pm 1,46$ & $0,86 \pm 0,69$ & $0,43 \pm 0,79$ & $1,00 \pm 1,53$ & $1,00 \pm 1,15$ & $0,14 \pm 0,38$ \\
\hline $\begin{array}{l}\text { Raiva- } \\
\text { Hostilidade }\end{array}$ & $0,14 \pm 0,38$ & 0 & $0,29 \pm 0,76$ & $1,14 \pm 1,86$ & $0,29 \pm 0,76$ & $0,57 \pm 1,51$ \\
\hline Vigor & $10,57 \pm 1,72$ & $10,29 \pm 2,21$ & $12,14 \pm 2,54$ & $9,71 \pm 2,69$ & $8,71 \pm 2,21$ & $9,29 \pm 2,50 \mathrm{a}$ \\
\hline Fadiga & $3,43 \pm 2,70$ & $3,86 \pm 4,10$ & $2,57 \pm 3,05$ & $9,00 \pm 2,65 \mathrm{a}$ & $8,57 \pm 3,41 \mathrm{a}$ & $7,43 \pm 3,41 \mathrm{a}$ \\
\hline $\begin{array}{l}\text { Confusão } \\
\text { Mental }\end{array}$ & $1,29 \pm 2,36$ & $0,86 \pm 1,07$ & $0,43 \pm 0,79$ & $1,57 \pm 1,57$ & $2,00 \pm 2,58$ & $1,14 \pm 1,77$ \\
\hline DTH & $-2,57 \pm 4,89$ & $-2,43 \pm 5,29$ & $-6,57 \pm 5,38$ & $5,29 \pm 6,10 \mathrm{a}$ & $5,71 \pm 6,55 \mathrm{a}$ & $2,71 \pm 7,99 \mathrm{a}$ \\
\hline \multirow{2}{*}{ Variáveis } & \multicolumn{3}{|c|}{$30^{\prime}$} & \multicolumn{3}{|c|}{$60^{\prime}$} \\
\hline & Verbal & Silêncio & Música & Verbal & Silêncio & Música \\
\hline $\begin{array}{l}\text { Tensão- } \\
\text { Ansiedade }\end{array}$ & $2,14 \pm 1,68$ & $0,86 \pm 1,07$ & $0,71 \pm 1,25 b$ & $1,43 \pm 1,51$ & $0,86 \pm 0,90$ & $2,00 \pm 1,73$ \\
\hline Depressão & $1,00 \pm 0,82$ & $0,14 \pm 0,38 \mathrm{bc}$ & $0,14 \pm 0,38 \mathrm{c}$ & $0,43 \pm 0,79$ & $0,29 \pm 0,49$ & $0,14 \pm 0,38$ \\
\hline $\begin{array}{l}\text { Raiva- } \\
\text { Hostilidade }\end{array}$ & $0,29 \pm 0,49$ & $0,57 \pm 1,13$ & 0 & 0 & 0 & 0 \\
\hline Vigor & $9,14 \pm 2,73$ & $9,43 \pm 3,10$ & $10,57 \pm 3,10$ & $10,57 \pm 3,82$ & $10,00 \pm 2,65$ & $10,57 \pm 3,60 \mathrm{a}$ \\
\hline Fadiga & $5,86 \pm 3,08 \mathrm{ab}$ & $5,57 \pm 2,76 b$ & $5,29 \pm 2,87 \mathrm{a}$ & $5,00 \pm 2,31 \mathrm{~b}$ & $5,57 \pm 2,37 \mathrm{~b}$ & $4,57 \pm 3,91 b$ \\
\hline $\begin{array}{l}\text { Confusão } \\
\text { Mental }\end{array}$ & $1,29 \pm 1,11$ & $0,71 \pm 0,95$ & $0 \mathrm{c}$ & $1,14 \pm 1,21$ & $0,57 \pm 1,13$ & $0 \mathrm{c}$ \\
\hline DTH & $1,43 \pm 3,51$ & $-1,57 \pm 2,82 b$ & $-4,43 \pm 3,9 b c$ & $-2,57 \pm 4,69 b$ & $-2,71 \pm 3,50 b$ & $-3,86 \pm 5,73 c$ \\
\hline
\end{tabular}

ANOVA two-way para medidas repetidas, com fatores Tempo e Grupo, com post-hoc utilizando Duncan Test. a - diferente do basal intra-grupo; b - diferente do imediatamente após intra-grupo; c - diferente do grupo Verbal no mesmo momento. Resultados significativos para $\mathrm{p} \leq 0,05$. Dados apresentados em média \pm desvio-padrão.

basal e ao momento imediatamente após. Para o grupo Silêncio, observamos na dimensão Ansiedade uma diminuição no momento imediatamente após, com aumentos comparados a esse momento 30 e 60 minutos. Diminuição em relação ao basal também foi observado na dimensão sedação física e OSA. Para o protocolo realizado com Música, a dimensão sedação física se mostrou diminuída em relação ao basal em todos os momentos avaliados. Na dimensão Sedação Mental observamos uma redução no momento 30 minutos após quando comparado ao basal. Nas outras variáveis não observamos diferenças significativas.

\section{Discussão}

Com o intuito de investigar a influência da motivação nas respostas afetivas após teste realizado até a exaustão voluntária máxima, submetemos um grupo de jovens a tipos de incentivos. Um dos grandes estímulos que despertou interesse para compor esse estudo, reside na compreensão da importância da motivação para a realização do exercício físico, sendo essa, possível de interferir no resultado final, isto é, executar níveis mais elevados de esforço (Andreacci et al., 2002). Nesse contexto, de forma geral, estar motivado enquanto se exercita, permite que ocorra um aumento na carga de trabalho, e uma série de efeitos psicofísicos que estão relacionados com redução da percepção subjetiva de esforço e aumento dos estados afetivos (Karageorghis, Terry \& Lane, 
Figura 1

\section{Escala Visual Analógica (VAMS)}

Basal = Imediatamente Após =30' =60'
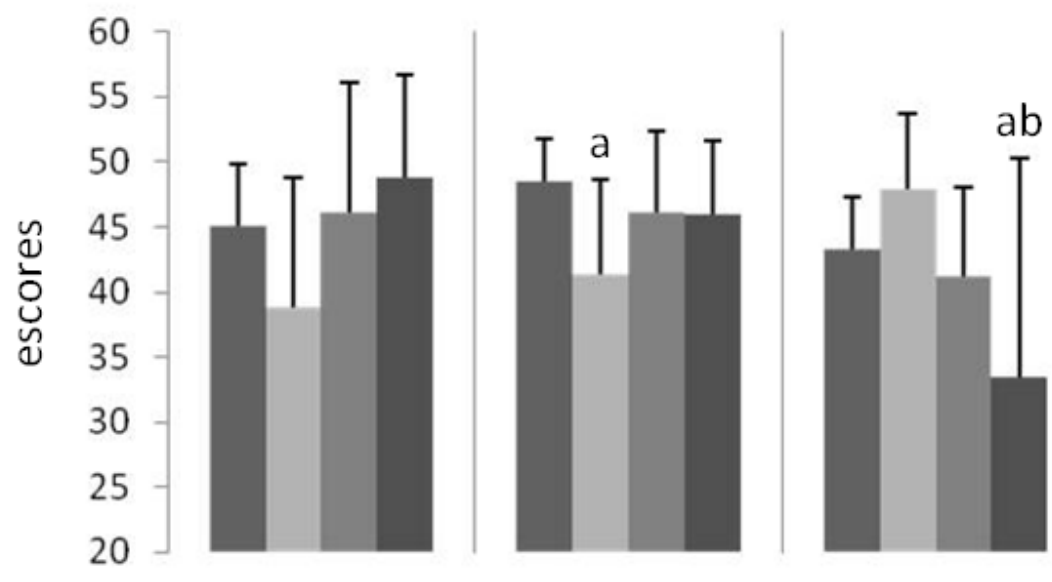

(A)
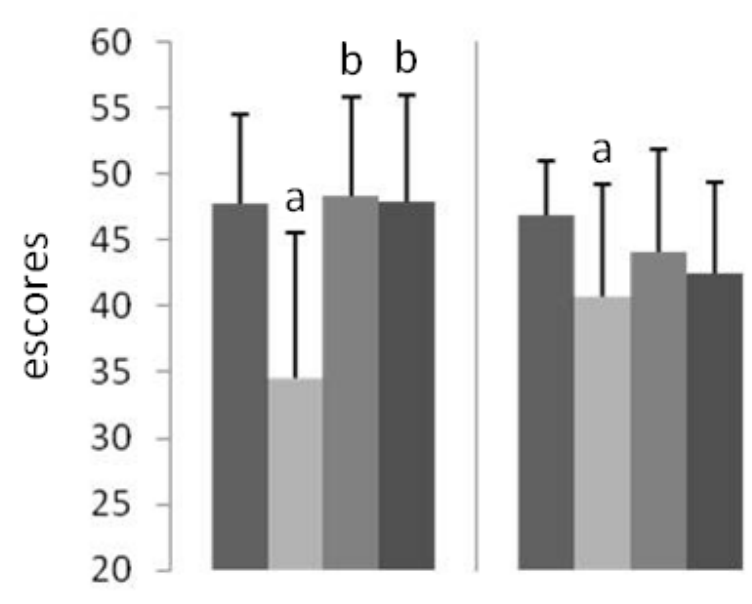

(B)

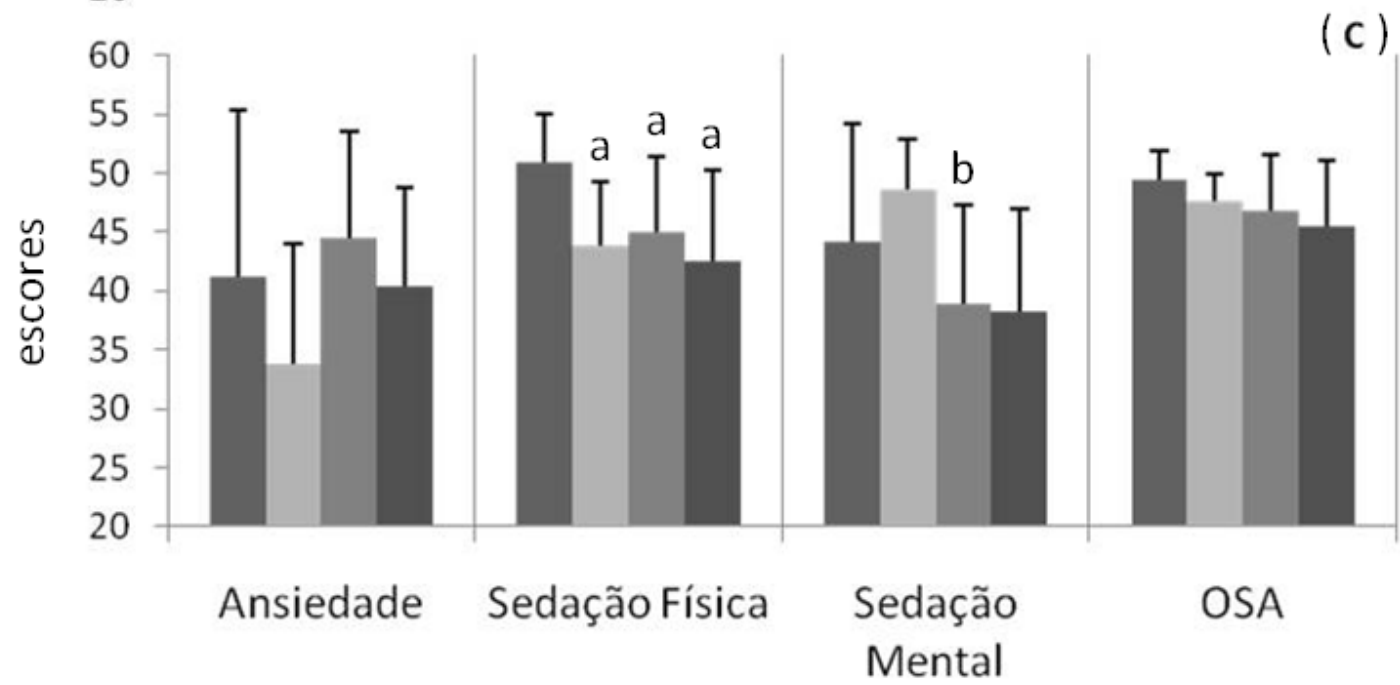

ANOVA para medidas repetidas, com post-hoc utilizando Duncan Test. a - diferente do basal intra grupo; $\mathrm{b}$ - diferente do imediatamente após intra grupo. Resultados significativos para $\mathrm{p} \leq 0,05$. Dados apresentados em média \pm desvio-padrão, onde: (A)- Verbal; (B) Silêncio; (C) Música. Legenda: OSA- Outros Sentimentos e Atitudes. 
1999). Assim, a motivação em um contexto ampliado, pode representar uma importante estratégia no sentido de melhorar o desempenho, caracterizando como um recurso ergogênico lícito e benéfico.

Em relação aos nossos achados, quando as variáveis ventilatórias e o comportamento da freqüência cardíaca (FC) dos diferentes protocolos foram comparados, não encontramos diferenças significativas em nenhuma das variáveis estudadas. Esse resultado vem de encontro aos achados de Schie, Stewart, Becker \& Rogers (2008), que também não encontraram modificações na resposta da freqüência cardíaca em um estudo onde homens e mulheres realizaram exercícios submáximos em cicloergômetro em duas condições, com e sem música. Nesse estudo, também foram analisados o consumo de oxigênio e a concentração de lactato no plasma, variáveis as quais também não apontaram nenhuma diferença significativa, porém, a maioria dos seus voluntários alegou que a sessão com música teria sido executada de forma mais fácil em relação à outra.

Por outro lado, um considerável número de trabalhos vem demonstrando sistematicamente que existem modificações nesta variável em protocolos que envolvem exercício físico com estímulo incentivador da música (Eliakim et al., 2007; Lim, Atkinson, Karageorghis \& Eubank, 2009; Karageorghis, Jones, Stuart, 2008). Eliakim et. al., (2007) analisou a influência da música durante o aquecimento de atletas de vôlei, evidenciando mudanças em relação à $\mathrm{FC}$ pico, a qual foi atingida antes quando comparada com o aquecimento sem música. No estudo de Aktison,Wilson \& Eubank (2004), foi analisado o comportamento da FC em ciclistas amadores, o diferencial do trabalho foi em relação à análise do tempo de percurso, onde os voluntários apresentaram diferenças no tempo total da execução, que foi menor na condição de música em contrapartida ao mesmo protocolo sem música. Em seu estudo, também foi encontrado diferenças nas variáveis cardíacas, as quais tiveram níveis aumentados nas condições de exercício quando executado com o incentivo da música, principalmente nos primeiros quilômetros quando comparado com a execução sem música.

É importante mencionar que a maioria dos trabalhos que investigaram esse binômio "música e exercício", focou essencialmente em protocolos submáximos o que de certa forma evidência $o$ ineditismo desse trabalho. Muito recentemente, Riganello, Candelieri, Quintieri, Conforti \& Dolce (2010), observou mudanças autonômicas em pacientes com danos cerebrais e controles saudáveis, quando esses escutaram uma lista de músicas préselecionadas. O interessante desse estudo, é que os autores mesmo que de forma preliminar, encontraram que essas mudanças são sensíveis a música, particularmente por esse tipo de estratégia permitir mudanças nos estados emocionais. Nesse estudo, diferente do nosso protocolo, foi utilizado músicas clássicas e condições de repouso, o que talvez tenha influenciado na diferença dos resultados.

Sobre as variáveis ventilatórias, acreditávamos que haveria diferenças nesses parâmetros, pois a motivação particularmente para realizar esforços de natureza máxima, é um adicional importante para obtenção de êxito nesse tipo de atividade, e quanto maior é esforço, maiores são as respostas ventilatórias, pois essas se modificam de forma linear com a intensidade (McArdle, Katch \& Katch, 1998; Powers \& Howley, 2000).

Nesse sentido, acreditávamos que os incentivos: Verbal e Música pudessem refletir nessas variáveis, no entanto isso não ocorreu. Embora as médias das variáveis do teste ergoespirométrico estivessem maiores do que o teste executado em Silêncio, esses não foram significativos, em parte esse resultado pode ser justificado pela amostra reduzida. Por outro lado, é possível que os incentivos utilizados não tenham sido suficientes para contribuir com a ativação mais enfática do sistema nervoso central, especificamente: Sistema Límbico - Tálamo - Hipotálamo - Hipófise - Adrenal, particularmente relacionado ao controle hormonal, onde durante uma atividade física até a exaustão voluntaria máxima, o indivíduo motivado pode sofrer alterações hormonais que o ajudará a manter, ou poderá até melhorar o estado físico e psicológico, com reflexo positivo no desempenho do atleta durante a prática da atividade. Desta forma, acreditamos que não tenha sido possível estabelecer uma relação de economia de esforço para uma mesma intensidade, nem mesmo um aumento do nível de alerta, ou decréscimo da fadiga com concomitante aumento da sincronização e coordenação motora (Karageoghis \& Terry, 1997; Vasconcelos, 1995).

No entanto, encontramos modificações intra e inter protocolos quando os aspectos psicobiológicos foram analisados. Assim, do ponto de vista psicobiológico, nossos resultados mostraram que no questionário BRUMS foi observado diferenças na dimensão Fadiga com comportamentos semelhantes nos três tipos de incentivo, apresentando escores aumentados nos momentos imediatamente após quando comparado com o basal, seguido de redução nos momentos seguintes. Este comportamento já era esperado, por se tratar de um protocolo de exercício até a exaustão máxima, no qual se supõe um empenho do voluntário para alcançar seu melhor desempenho possível, o que consequentemente geraria sintomas de Fadiga. Nahas (2001) define de um modo geral esse estado, como um conjunto de alterações que ocorre no organismo, resultantes de atividades físicas ou mentais e que levam a uma sensação generalizada de cansaço sendo consequência direta da fadiga a perda de eficiência, ou seja, a diminuição da capacidade de trabalho.

Ainda nesse questionário, mas na dimensão DTH (Distúrbio total de humor), foi observado um 
aumento dos escores no momento imediatamente após, seguido de redução nos momento posteriores. $\mathrm{O}$ DTH representa um parâmetro de análise generalista do humor, pois ele reflete a soma de todas as dimensões medidas por esse questionário menos a dimensão vigor. Desta forma, observamos que ao final do exercício, houve uma alteração do humor que foi restabelecida entre 30 e 60 minutos do término dessa atividade. Porém, comparando essas respostas entre os tipos de incentivo, podemos observar que no protocolo que envolveu Encorajamento Verbal o momento imediatamente após está aumentado quando comparado com o basal, mas, este se mantém aumentado no momento $30^{\prime}$ e voltando aos níveis basais apenas no momento 60', um comportamento diferente dos outros tipos de incentivo, os quais tiveram seu retorno para os níveis basais já no momento 30', o que aparentemente sugere que os incentivos com Música e Silêncio interfiram menos nas alterações de humor ou que promovam um retorno mais rápido nas alterações sofridas. Ainda sim, no incentivo com Música, mesmo que não significativo estatisticamente, este apresentou um aumento menor em relação aos outros incentivos no momento imediatamente após, o que poderia demonstrar indícios de que a música interfira ainda menos nas alterações do humor.

$\mathrm{Na}$ dimensão Vigor os grupos exibiram comportamento similar, no entanto tal comportamento só foi significativo no protocolo realizado com Música, onde após o exercício, esse grupo apresentou menores escores no momento imediatamente após e 60' após quando comparado ao basal. Esse resultado sugere que 60' após o término do exercício não foram suficientes para recuperar essa dimensão, nessa linha de raciocínio, é possível que a fadiga gerada nesse protocolo tenha sido assimilada do ponto de vista psicobiológico como extrema, e mesmo que o DTH tenha se recuperado parcialmente, a dimensão Vigor não apresentou comportamento similar.

No questionário VAMS, a dimensão Sedação Física apresentou respostas com diferenças semelhantes nos três tipos de estímulo. O Encorajamento Verbal e o Silêncio apresentaram escores menores no momento imediatamente após quando comparado com o basal, entretanto, no incentivo com Música este escore ainda permaneceu reduzido nos momentos seguintes quando comparados com o basal. Esse comportamento pode apontar indícios de que a música como incentivo poderia interferir de forma mais positiva no humor mantendo seus efeitos por mais tempo.

Ainda, no questionário VAMS, a dimensão Ansiedade apresentou diferenças somente no protocolo executado em Silêncio, observamos redução desse escore apenas no momento imediatamente após quando comparado com o basal. Esse comportamento era esperado nos três diferentes protocolos, afinal, existe um consenso entre diversos autores de que o exercício físico contribui na minimização dos sintomas de ansiedade, promovendo assim efeitos ansiolíticos após sua prática, causando benefícios na redução desses níveis, sendo claro o papel do exercício físico como uma importante estratégia de intervenção não farmacológica nas alterações de humor (Wipfli, Rethorst \& Landers, 2008; Morgan, 1970; Stella, Antunes, Santos, Galduróz \& de Mello, 2004; Petruzzello, Hall \& Ekkekakis, 2001).

Entretanto, talvez esse comportamento tenha sido influenciado pela preferência do tipo de música. Nesse contexto, o Silêncio pode ter sido mais eficaz na resposta ansiolítica por ser neutro quanto à preferência quando comparado com a música ou com o incentivo verbal, os quais podem influenciar negativamente, se tornando um fator estressante por carregarem relações emocionais e quanto à preferência. Estudos apontam a música em uma frequência (bpm) mais rápida como a mais adequada para o exercício vigoroso, devido ao aumento da excitação fisiológica associada (Edworthy, Waring, 2006; Priest, Karageorghis, Sharp, 2004). Ainda nessa linha, Szabo, Small \& Leigh, (1999), mostram que a exposição contínua e prolongada a músicas de frequências rápidas e ou alta possam resultar em consequências negativas, tais como queda da motivação, tédio e a irritação e, além disso, que uma sequência de músicas de tempo misto possa ter efeitos motivacionais positivos maiores quando comparado com uma sequência de ritmo único. Nosso estudo não avaliou a preferência musical dos voluntários, o que talvez possa ter interferido nas respostas psicobiológicas dos mesmos, influências as quais destacam Becker, et al. (1994) e Pujol \& Langenfeld (1999) que corroboram suas evidências sobre a investigação dos efeitos da música e a sua relação com desempenho físico e afirmam que os resultados da literatura em geral são conflitantes, pois sugerem que o tempo, o tipo de música, o tipo de exercício e o nível de condicionamento físico do atleta afetariam na resposta de desempenho frente à música.

$\mathrm{Na}$ dimensão Sedação Mental, do mesmo questionário, foi possível observar alterações significativas no protocolo com Encorajamento Verbal, o qual teve um escore menor quando comparado com os momentos basal e imediatamente após e no protocolo com Música que apresentou uma queda no seu escore também no momento 60 ' quando comparado com o momento imediatamente após. A dimensão sedação mental contrapõe estados de alerta, sonolência, atenção e distração, sendo assim, esses resultados podem sugerir que o impacto do protocolo com Encorajamento Verbal seja maior nessas variáveis, pois nenhum dos extremos desses estados é positivo para o indivíduo apontando assim que talvez o Silêncio seja capaz de manter esses estados de humor mais normalizados do que os outros protocolos.

Os resultados da escala subjetiva de experiência 
de exercício (SEES) apontaram diferenças nas três dimensões e nos diferentes incentivos. Sendo que, a dimensão fadiga manteve o mesmo comportamento dos outros questionários discutidos anteriormente, se mantendo alta no momento pós-exercício e reduzindo nos momentos seguintes.

No mesmo questionário, observamos que o comportamento da dimensão bem estar positivo apresentou diferenças no momento imediatamente após dos três incentivos, os quais apresentam uma queda no momento imediatamente após quando comparado com os valores basais e um retorno progressivo aos mesmos. Embora apenas o protocolo com Encorajamento Verbal tenha apresentado diferenças significativas, o que talvez indique sua influência para um retorno mais eficaz aos níveis basais, os outros incentivos também seguiram uma mesma linha de progressão. Reed (2005) e Kwan \& Bryan (2009), neste contexto, mostram que sessões agudas de exercício podem levar a uma melhoria imediata no afeto positivo e negativo, gerando respostas positivas no comportamento, e talvez mais importantes, manter a motivação para o exercício.

A dimensão distresse psicológico, nesse questionário, apresentou diferenças similares no protocolo com Incentivo Verbal e em Silêncio. Ambos aumentaram seus escores no momento imediatamente após e recuperaram esses valores no momento 60', porém o protocolo com Música apresentou um comportamento linear em todos os momentos do protocolo. Jekel, Elmore, Katz \& Jekel (1999), definem distresse psicológico como uma incapacidade para superar a vivência de experiências estressantes, que desgastam o indivíduo, levando a uma ruptura do bem-estar individual. Sendo assim, esses resultados sugerem uma influência positiva da música como elemento incentivador, pois essa foi capaz de amenizar um possível impacto psicológico negativo gerado pelo exercício até a exaustão, como citam Ekkekakis e Petruzzello (1999) que sugerem que a prática do exercício físico acima do limiar gera uma situação de perturbação homeostática severa do organismo refletindo assim em respostas emocionais negativas e a predominância de fatores somáticos.

No questionário IDATE os resultados dos protocolos apontaram um comportamentos similares, no qual observamos uma resposta ansiogênica do exercício principalmente no momento imediatamente após, porém, com uma significativa redução desses escores nos momentos seguintes. Esses resultados foram contra os achados de ansiedade mensurados pelo questionário VAMS o qual mostra uma queda dos níveis de ansiedade logo após o exercício, porém é necessário ressaltar que segundo Guimarães, et. al.(1998), que definem o IDATE como um questionário específico de auto avaliação da ansiedade, diferente do VAMS que sugere indicativos nas alterações do humor em geral. Mesmo assim, destaca ambos como capazes de apontar o comportamento dessa variável, e nesse contexto, sugere como uma atitude prudente o emprego em um mesmo estudo dessas diferentes escalas. Os resultados do IDATE apontaram um efeito ansiogênico no momento imediatamente após, porém uma redução desses níveis nos momentos seguintes, o que também vai de encontro com a literatura citada anteriormente.

Nas análises comparativas entre os grupos observamos diferenças apenas no questionário BRUMS, que apontou um comportamento distinto entre os grupos em relação à dimensão depressão, confusão mental e no DTH. A dimensão depressão foi diferente no momento 30' do grupo com Encorajamento Verbal quando comparado com o grupo que recebeu incentivo com a Música e Silêncio, já a confusão mental do protocolo com encorajamento também foi diferente, neste momento e aos 60', do grupo que realizou o protocolo em Silêncio. Por último, observamos alterações na dimensão DTH, no momento 30', quando comparado protocolo com Encorajamento Verbal em relação ao realizado com a música. Com esses resultados, observamos principalmente diferenças no protocolo com Encorajamento Verbal em relação aos outros. Este aparenta interferir mais negativamente do que os outros protocolos, onde é possível que o tom de voz e as palavras de incentivo possam ser desagradáveis aos voluntários, assim seria conveniente questionar o voluntário, antes de realizar um teste dessa natureza, se o mesmo se incomoda em ouvir palavras de encorajamento durante o teste.

Por fim, considerando a literatura analisada e os resultados encontrados, nosso estudo avança no que diz respeito à relação motivação, seja por Encorajamento Verbal, Música ou até mesmo pelo Silêncio na compreensão das influências destes na exaustão voluntaria máxima. Isso parece ser importante, pois durante o exercício máximo e até mesmo supra-máximo, atletas expressam seus sentimentos que variam de fadiga até exaustão. Estar motivado pelo encorajamento ou pela música distrai o atleta de sensações de fadiga associados ao exercício por meio de dissociação (Karageorghis, Terry \& Lane, 1999; Rejeski, 1985; Andreacci, et al., 2002), permitindo assim uma possível melhora.

\section{Conclusão}

Considerando os dados observados, o presente estudo permite concluir que a motivação interfere de forma mais evidente nos aspectos psicobiológicos de humor e bem estar do que nas respostas fisiológicas. O estudo mostrou que os diferentes incentivos podem ser usados como estratégias eficazes no desempenho físico dependendo do objetivo proposto, não sendo seus benefícios ou prejuízos limitados ou restritos a 
apenas um tipo de incentivo, tendo os três tipos suas particularidades.

\section{Referências}

Aktison, G., Wilson, D., \& Eubank, M. (2004). Effects of Music on Work-Rate Distribution During a Cycling Time Trial. International Journal of Sports Medicine, 25, 611-615.

Andreacci, J. L., LeMura, L. M., Cohen, S. L., Urbansky, E. A., Chelland, S. A., \& Von Duvillard, S. P. (2002). The effects of frequency of encouragement on performance during maximal exercise testing. Journal of Sports Sciences, 20, 345-352.

Andreatini, R., \& Seabra, M. L. (1993). A estabilidade do IDATE-traço: avaliação após cinco anos. Revista ABP-APAL, 15 (1), 21-25

Becker, N., Brett, S., Chambliss, C., Crowers, K., Haring, P., Marsh, C., \& Montemayor, R. (1994). Mellow and frenetic antecedent music during athletic performance of children, adults, and seniors. Perceptual \& Motor Skills, 79, 1043-1046.

Biaggio, A. M. B., \& Natalicio, L. (1979). Manual para o inventário de ansiedade traço-estado (Idate). Rio de Janeiro: Centro Editor de Psicologia Aplicada CEPA.

Bond, A., \& Lader, M. (1974). The use of analogue scales in rating subjective feelings. British Journal of Psychology, 47, 211218.

Copeland, B. L., \& Franks, B. D. (1991). Effects of types and intensities of background music on treadmill endurance. The Journal of Sports Medicine and Physical Fitness, 31, 100-103.

Edworthy, J., \& Waring, H. (2006). The effects of music tempo and loudness level on treadmill exercise. Ergonomics, 49, 15971610 .

Ekkekakis, P., \& Petruzzello, S. J. (1999). Acute aerobic exercise and effect: current status, problems and prospects regarding dose-response. Sports Medicine, 28 (5), 337-374.

Eliakim, M., Meckel, Y., Nemet, D., \& Eliakim, A. (2007). The effect of music during warm-up on consecutive anaerobic performance in elite adolescent volleyball players. Int J Sports Medicine, 28, 321-325.

Ferguson, A. R., Carbonneau, M. R, \& Chambliss C. (1994). Effects of positive and negative music on performance of a karate drill. Perceptual \& Motor Skills, 78, 1217-1218.

Guimarães, F. S., Zuardi, A. W., Gentil, V., \& Graeff, F. G. (1998). A Importância do treinamento Prévio em Escala Analógica de Auto-avaliação. Revista ABAP-APAL, 10(1), 5-7.

Jekel, J. F., Elmore, J. G., \& Katz, D. L. (1999). Introdução à medicina preventiva. In: Jekel, J. F., Elmore, J. G., \& Katz, D. L., Epidemiologia, bioestatística e medicina preventiva (pp. 203-209). Porto Alegre: Artes Médicas Sul.

Karageorghis C.I., Drew K.M., \& Terry P.C. (1996). Effects of pretest stimulative and sedative music on grip strength. Perceptual \& Motor Skills, 83, 1347-1352.

Karageorghis, C. I., \& Terry, P. C. (1997). The psychophysical effect of music in sport and exercise: a review. Journal of Sport Behavior, 20, 54-68.

Karageorghis, C. I., Terry, P. C., \& Lane, A. M. (1999). Development and initial validation of an instrument to assess the motivational qualities of music in exercise and sport: The Brunel Music Rating Inventory. Journal of Sports Science, 17, 713-724.

Karageorghis, C., Jones, L., \& Stuart, D. P. (2008). Psychological effects of music tempi during exercise. International Journal of Sports Medicine, 29 (7), 613-9.

Kwan, B. M., \& Bryan, A. D. (2009). Affective response to exercise as a component of exercise motivation: Attitudes, norms, self-efficacy, and temporal stability of intentions. Psychology of Sport and Exercise, 11, (1), 71-79.

Lim, H. B. T., Atkinson, G., Karageorghis, C. I., \& Eubank, M. M. (2009). Effects of differentiated music on cycling time trial. International Journal of Sports Medicine, 30, 435-442.

Lox, C. L., \& Rudolph, D. L. (1994). The subjective exercise experiences scale (SEES): factorial validity and effects of acute exercise. Journal of Social Behavior and Personality, 9, 837-844.
McArdle, W. D., Katch, F. I., \& Katch, V. R. (1998). Fisiologia do exercício: energia, nutrição e desempenho humano. Rio de Janeiro: Guanabara Koogan.

Mcauley, E., \& Courneya, K. (1994). The Subjective Exercise Experiences Scale (SEES): Development and preliminary validation. Journal of Sport \& Exercise Psychology, 16, 163-177.

Mcnair, D. M., Lorr, M., \& Droppleman, L. F. (1971). Profile Mood States: Manual. San Diego: Educational and Industrial Testing Services.

Morgan, W. P. (1970). Physical working capacity in depressed and non-depressed females: A preliminary study. American Corrective Therapy Journal, 24, 14-26.

Nahas, M. V. (2001). Atividade física, saúde e qualidade de vida: conceitos e sugestões para um estilo de vida ativo. Londrina: Midiograf.

Petruzzello, S. J., Hall, E. E., \& Ekkekakis, P. (2001). Regional brain activations as a biological marker of affective responsity to acute exercise: influence of fitness. Psychophysiology, 38, 99 -106.

Powers, S. K., \& Howley, E. T. (2000). Fisiologia do exercício: teoria e aplicação ao condicionamento e ao desempenho. São Paulo: Manole.

Priest, D. L., Karageorghis, C. I., \& Sharp, N. C. C. (2004). The characteristics and effects of motivational music in exercise settings: the possible influence of gender, age, frequency of attendance, and time of attendance. The Journal of Sports Medicine and Physical Fitness, 44, 77-86.

Pujol, T. J., \& Langenfeld, M. E. (1999). Influence of music on Wingate Anaerobic Test performance. Perceptual \& Motor Skills, 88, 292-296.

Reed, J. (2005). Acute physical activity and self-reported affect: a review. In: Clark, A. V. Causes, role and influence of mood states (pp. 91-113). Chicago: Nova Science Publishers.

Rejeski, W. J. (1985) Perceived exertion: an active or passive process. Journal of Sport Psychology, 7, 371-378.

Riganello, F., Candelieri, A., Quintieri, M., Conforti, D., \& Dolce, G. (2010). Heart rate variability: An index of brain processing in vegetative state? An artificial intelligence, data mining study. Clinical Neurophysioly, 121(12), 2024-34.

Rogol, A. D. (2003). Dietary supplements to enhance athletic performance. In: Lifshitz F. (Ed.), Pediatric Endocrinology. (4 ed., pp. 917-922). New York: Marcel Dekker.

Rohlfs, I. C., Carvalho, T. D., \& Rotta, T. M. (2004). Aplicação de instrumentos de avaliação de estados de humor na detecção da síndrome do excesso de treinamento. Revista Brasileira de Medicina do Esporte 2, 111-116.

Sassi, A., Marcora, S. M., Rampinini, E., Mognoni, P., \& Impellizzeri, F. M. (2006) Prediction of time to exhaustion from blood lactate response during submaximal exercise in competitive cyclists. European Journal of Applied Physiology, 97, 174-180.

Schie, N. A., Stewart, A., Becker, P., \& Rogers, G. G. (2008). Effect of music on submaximal cycling. South African Journal of Sports Science, 20, 28-31.

Spielberg, C. D., Gorshusch, R. L., \& Lushene, E. (1970). Manual for the State-Trait Anxiety Inventory: Self-Evaluation Questionnaire. Palo Alto, CA.: Consulting Psychologist Press

Stella, S. G., Antunes, H. K. M., Santos, R. F., Galduróz, J. C. F., \& de Mello, M. T. (2004). Transtornos do Humor e Exercício Físico. In: de Mello, M. T., \& Tufik, S. Atividade Física, Exercício Físico e Aspectos Psicobiológicos. (pp. 51-55). São Paulo: Guanabara Koogan.

Szabo, A., Small, A., \& Leigh, M. (1999). The effects of slowand fast-rhythm classical music on progressive cycling to voluntary physical exhaustion. J Sports Med Phys Fit, 39, 220-225.

Szmedra, L., \& Bacharach, D. W. (1998). Effect of music on perceived exertion, plasma lactate, norepinephrine and cardiovascular hemodynamics during treadmill running. International Journal of Sports Medicine, 19, 32-37.

Terry, P. C., Lane, A. M., \& Forgaty, G. J. (2003). Construct validity of the POMS-A for use with adults. Psychology of Sports and Exercise, 4, 125-39.

Vasconcelos, E. G. (1995). O prazer e a dor do corpo em estresse. São Paulo: Instituto de Psicologia da USP. 
Wasserman, K. (1987). Determinants and detection of anaerobic threshold and consequences of exercise above it. Circulation, 76, 29-39.

Wasserman, K., \& Koike, A. (1992). Is the anaerobic threshold truly anaerobic? Chest, 101, 211-218.

Wipfli, B .M., Rethorst, C. D, \& Landers, D. M. (2008). The anxiolytic effects of exercise: a meta-analysis of randomized trials and dose-response analysis. Journal of Sport \& Exercise Psychology, 30, 392-410.

Yamamoto, T., Ohkuwa, T., Itoh, H., Kitoh, M., Terasawa, J., Tsuda, T., Kitagawa, S., \& Sato, Y. (2003). Effects of preexercise listening to slow and fast rhythm music on supramaximal cycle performance and selected metabolic variables. Archives of Physiology and Biochemistry, 111, 211-214.

Recebido: 06/12/2010

Última Revisão: 12/09/2011

Aceite Final: 26/10/2011

Agradecimentos

Os autores agradecem o apoio científico de Everald Vancouler e Natasha Mayerhofer e o apoio técnico e financeiro da AFIP, CEPE, CEMSA, CEPID-SONO/FAPESP (processo número: 1998/14303-3), CNPq, INSTITUTO DO SONO e a FADA/UNIFESP. 